\title{
Minimal Pole Figure Ranges for Quantitative Texture Analysis
}

\author{
K. Helming \\ JINR Dubna, Head Post Office P.O. Box 79, \\ 101000 MOSCOW, USSR
}

\begin{abstract}
The use of only a small number of incomplete pole figures for texture determinations is of practical interest for reducing the effort of texture measurement. The determination of minimal pole figure ranges (MPR) is explained and the use of MPR is demonstrated on an example.
\end{abstract}

\section{Reproduction Problem}

During the last years the quantitative texture analysis (QTA) has been more and more established in its traditional field of metal research as well as in geosciences and other applied fields. Polycrystalline samples are investigated consisting of some crystalline phases which may possess low crystal symmetry. The texture of a polycrystalline phase will be described quantitatively by the orientation distribution function (ODF) $f(g)$

$$
\int_{G} f(g) d g=8 \pi^{2}, \quad d g=\sin \alpha d \alpha d \beta d \gamma, \quad f(g) \geq 0 .
$$

with $G: 0 \leq \alpha, \gamma<2 \pi, 0 \leq \beta \leq \pi$. At this $\{\alpha, \beta, \gamma\} \equiv g$ are three Eulerian angles describing the orientation of two right-handed cartesian coordinate systems relatively to each other.

Starting points for the calculation of the ODF are mostly diffraction patterns (X-ray, thermal neutrons, electrons). From this so called reduced pole figures (PF)

$$
\begin{aligned}
\tilde{P}_{\vec{h}_{i}}\left(\vec{y}_{i}\right) & =\left[P_{\vec{h}_{i}}\left(\vec{y}_{i}\right)+P_{-\vec{h}_{i}}\left(\vec{y}_{i}\right)\right] / 2, \\
\text { with } \quad P_{\vec{h}_{i}}\left(\vec{y}_{3}\right) & =\frac{1}{2 \pi} \int_{\tilde{\varphi}_{i}=0}^{2 \pi} f\left(\left\{\vec{h}_{i}, \tilde{\varphi}_{i}\right\}^{-1}\left\{\vec{y}_{i}, 0\right\}\right) d \tilde{\varphi}_{i} .
\end{aligned}
$$

are determined. The number of normals of net planes $\vec{h}_{i}, i=1, \ldots I$ "visible" for the experiment and the quantity of measured pole directions $\vec{y}_{:}, l=1, \ldots L(i)$ is restricted by manifold experimental conditions. Under all circumstances it is neccessary to know are the measured data sufficient for QTA. Moreover the knowledge of the minimal expense of measurement i.e. of the minimal pole figure ranges (MPR) is important for optimizations of texture measurements. 
Starting only from principles of crystal geometry the determinaton of MPR is demonstrated in this paper. As an example the modelized ODF of a olivin sample is reproduced from such MPR using a new (geometrical) approach to QTA $^{1}$.

\section{Determination of a Single Orientation}

Crystal symmetry and normal scattering effect that all crystal directions

$$
\vec{h}_{i}^{j}=\tilde{g}_{b_{j}}^{-1} \vec{h}_{i}, \quad \tilde{g}_{b_{j}}, \in \tilde{\mathcal{G}}_{B}=\mathcal{C}_{i} \times \mathcal{G}_{B}, j^{\prime}=1 \ldots \tilde{N}_{b}, j=1 \ldots J\left(\tilde{\mathcal{G}}_{B}, \vec{h}_{i}\right)
$$

are equivalent in regard to the Laue group $\tilde{\mathcal{G}}_{B}=\mathcal{C}_{i} \times \mathcal{G}_{B}\left(\mathcal{G}_{B}-\right.$ crystal class), i.e. we are not able to distinguish between them. The number of all equivalent but not parallel directions $\tilde{h}_{i}^{j}$ is given by $J\left(\tilde{\mathcal{G}}_{B}, \vec{h}_{i}\right)$. On the other hand all orientations $g_{B}, g$ given by the rotational part $G_{B}\left(g_{B}, \in G_{B}\right)$ of the crystal class $\mathcal{G}_{B}$ are equivalent too ${ }^{3}$. To determine $g_{B_{j}}, g$ the equation system

$$
\left(\vec{y}_{1}, \vec{y}_{2}, \vec{y}_{3}\right)=\left(g_{B_{j}}, g\right)^{-1}\left(\vec{h}_{1}^{j_{1}}, \vec{h}_{2}^{j_{2}}, \vec{h}_{3}^{j_{3}}\right) \quad g_{B_{i}}, \in G_{B}\left(\mathcal{G}_{B}\right)
$$

must be solved. It seems to be difficult to find out all conditions for the uniquness of the solution in an analytical manner ${ }^{2}$. Another "computational" approach is based on the following condition of the invariancy of correlation and uniqueness (CICU, sign $\stackrel{c u}{\Longleftrightarrow}$ ):

The orientation $g_{B}, g$ may be found uniquely if all sets of net planes $\left( \pm \vec{h}_{1}, \vec{h}_{2}^{j_{2}}, \vec{h}_{3}^{j_{3}}\right)$ possessing the same correlation as the set $\left(\vec{h}_{1}, \vec{h}_{2}, \vec{h}_{3}\right)$ (or its poles $\left(\vec{y}_{1}, \vec{y}_{2}, \vec{y}_{3}\right)$ ) may be transferred into each other by pure rotations $g_{B}, \in G_{B}$ only.

With the help of a computer program (called UNIQUE) this contition (CICU) may be proved for all Laue groups $\tilde{\mathcal{G}}_{B}=\mathcal{G}_{B} \times C_{i}$. Starting from a arbitrary set of three (or two) net planes $\left(\vec{h}_{1}, \vec{h}_{2}, \vec{h}_{3}\right)$ all sets $\left( \pm \vec{h}_{1}, \vec{h}_{2}^{j_{2}}, \vec{h}_{3}^{j_{3}}\right)$ possessing the same correlation are determined and are tested if they may be transferred into $\left(\vec{h}_{1}, \vec{h}_{2}, \vec{h}_{3}\right)$ by a rotational element $g_{B_{i}} \in G_{B}$. That means the program checks the CICU

$$
\left(\vec{h}_{1}, \vec{h}_{2}, \vec{h}_{3}\right) \stackrel{c u}{\Longleftrightarrow}\left( \pm \vec{h}_{1}, \vec{h}_{2}^{j_{2}}, \vec{h}_{3}^{j_{3}}\right) \quad j=1 \ldots J\left(\tilde{\mathcal{G}}_{B}, \vec{h}_{i}\right)
$$

for providing one equivalent solution $g_{B_{j}}, g$. One ore more $\vec{h}_{i}$ have to be exchanged if the CICU is not satisfied.

\section{Necessary Condition For QTA}

I pole figures $\tilde{P}_{\vec{h}_{i}}(\vec{y})(i=1 \ldots I)$ may be given in the pole figure ranges $Y_{i}$ to determine $f(g)$. The $Y_{i}$ could have a complex form, but mostly they are described by simple zones or calottes of the pole sphere. Considering the inversion symmetry caused by the normal scattering we use only one half sphere for the descripton the $Y_{i}$.

Following the conclusions of section we postulate a necessary condition for the QTA: 
Starting from I pole figures $\tilde{P}_{\vec{h}_{i}}(\vec{y})$ the ODF may be recalculated only if all poles $\vec{y}_{m}=g^{-1} \vec{h}_{i}^{j}$ with $\vec{y}_{m} \in Y_{i}, m=1 \ldots M(g)$ are sufficient for an unique determination of every orientation $g \in G$ (or $\left.g_{B}, g\right)$.

With other words the equation system

$$
\left(\ldots, \vec{y}_{m}, \ldots\right)=\left(g_{B_{j}}, g\right)^{-1}\left(\ldots, \vec{h}_{i_{m}}^{j_{m}}, \ldots\right)
$$

must satisfy the CICU $\left(\ldots, \vec{y}_{m}, \ldots\right) \stackrel{c u}{\Longleftrightarrow}\left(\ldots, \vec{h}_{i_{m}}^{j_{m}}, \ldots\right)$ for each $g \in G$. Using the program UNIQUE this can be proved for a given set of $Y_{i}$ in a simple manner.

\section{Minimal Pole Figure Ranges}

For optimizing texture measurements it is neccesary to find MPR satisfying CICU. Starting from only one pole figure these ranges were calculated by Vadon for the reflection case of scattering and called minimal pole density sets MPDS ${ }^{4}$. Their ranges are described by $\vartheta_{i}^{R}$ with $\vec{y}=\left(\vartheta_{y}, \varphi_{y}\right) \in Y_{i}, 0 \leq \vartheta_{y} \leq \vartheta_{i}^{R}$. For the transition case we have $\vartheta_{i}^{T} \leq \vartheta_{y} \leq \pi / 2$ (see ${ }^{2}$ ). MPDS only may be found for a small set of net planes and crystal classes (cubic, hexagonal, tetragonal). For determining the more general MPR $Y_{i}^{*}$ the following statement may be used:

For the case of triclinic sample symmetry all MPR $Y_{i}^{*}$ have to be symmetrical to a sample fixed axis $\vec{n}$, that means they always appear in form of zones or calottes on the pole sphere possessing a common centre given by $\vec{n}$.

Therefore the calculation of MPR is confined to the calculation of the range of the latitude $\vartheta_{y}$

$$
\vartheta_{s} \leq \vartheta_{y} \leq \vartheta_{e}, \vartheta_{y} \leq \pi / 2
$$

with

$$
\vartheta_{y}=\angle\left(\vec{g}^{*}, \vec{h}\right), \vec{g}^{*}=(\beta, \pi-\gamma)
$$

Commonly one of the limits $\vartheta_{s}$ or $\vartheta_{e}$ is given by the conditions of the experiment (e.g. $\vartheta_{s}=0$ for reflection, $\vartheta_{e}=\pi / 2$ for transmission measurement, cf. fig.1). The determination of the other limit for all possible $\vec{g}^{*}$ can be done in a numerical manner using (8).

\section{An EXAMPLE}

We consider a polycrystalline sample of olivin (crystal class $\mathcal{G}_{\mathcal{B}}=D_{2 h}$, lattice parameter relations $c / a=5.99 / 4.76$ and $c / b=5.99 / 10.21)$. Its texture shall be measured in reflection arrangement with $\vartheta_{s}=0$. Eight Bragg reflections may be analysed considered in six pole figures:
1. (021)
2. (101)
3. (111)/(120)
4. $(002) /(121)$
5. (130)
6. (112).

The third and fourth pole figure both include two closed together reflections. The corresponding normals of net planes $\vec{h}_{i}$ and it's equivalent directions are shown in regard to $K_{B}$ in Fig.1. We computed the latitudes $\vartheta_{y}$ for all possible $\vec{g}^{*}$ to determine the extremal values $\vartheta_{e}\left(\Delta \vec{g}^{*} \approx 1.5^{\circ}\right)$ : 


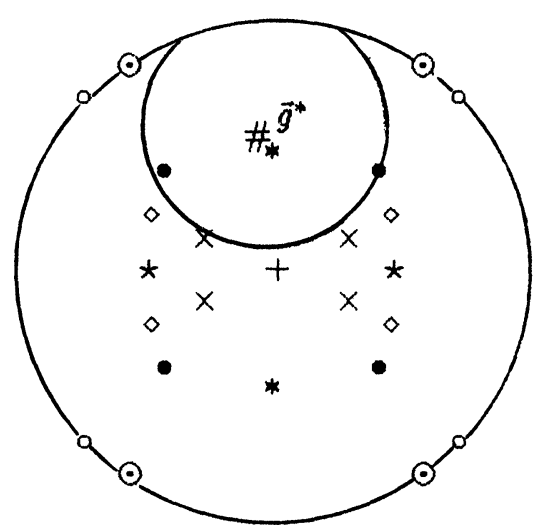

The arrangement of the eight net planes $\vec{h}_{1}\left(\vec{x}_{B}\right.$ points to the right, $\vec{y}_{B}$ upward) of the described example:

$$
\begin{array}{lll}
*-(021) & \star-(101) & \diamond-(111) \\
\circ-(120) & +-(002) & \bullet-(121) \\
\odot-(130) & \times-(112) &
\end{array}
$$

Within the plotted circle (radius $44.5^{\circ}$, centre \# at $\left.\vec{g}^{*}=\left(55^{\circ}, 96.1^{\circ}\right)\right)$ the $\vec{h}_{i}=(021),(121)$ are situated which are sufficient for the unambiguous determination of all orientations : $g=\left\{\ldots, 55^{\circ}, 83.9^{\circ}\right\}$.

Figure 1: Computation of MPR

\begin{tabular}{|c|c|c|c|c|c|c|c|c|}
\hline hkl & 021 & 101 & 111 & 120 & 002 & 121 & 130 & 112 \\
\hline$\vartheta_{e}$ & 40.8 & 40.5 & 41.1 & 42.1 & 38.0 & 44.5 & 43.8 & 43.8 \\
\hline
\end{tabular}

The maximum of $44.5^{\circ}$ is provided by the (121)-reflection of the fourth PF and may be found at $\vec{g}^{*}=\left(55^{\circ}, 96.1^{\circ}\right)$. Within the plotted circle (radius $44.5^{\circ}$, centre $\left.\vec{g}^{*}\right)$ in fig.1 the directions $\vec{h}_{i}=(021),(121)$ are situated which are sufficient for the unambiguous determination of all orientations : $g=\left\{\ldots, 55^{\circ}, 83.9^{\circ}\right\}$.

By means of Gauss and Lorentz shaped standard functions ${ }^{s} f_{k}^{G}, f_{k}^{L}, \tilde{P}_{k}^{G}$, $\tilde{P}_{k}^{L}$ an olivin-ODF consisting of two components

$$
\begin{gathered}
f(g)=\sum_{k}^{K=2}\left[G_{k} f_{k}^{G}\left(g_{k}, b_{k}\right)+L_{k} f_{k}^{L}\left(g_{k}, b_{k}\right)\right]+F \\
G_{k}+L_{k}=1, \int f(g) d g=8 \pi^{2}
\end{gathered}
$$

and the corresponding diffraction pole figures

$$
\begin{aligned}
\tilde{D}_{j}(\vec{y})= & n_{j}\left(\sum_{i}^{I} q_{i j} \sum_{k}^{K=2}\left[G_{k} \tilde{P}_{k}^{G}\left(g_{k}, b_{k}, \vec{h}_{i}\right)+L_{k} \tilde{P}_{k}^{L}\left(g_{k}, b_{k}, \vec{h}_{i}\right)\right]+F\right), \\
& \sum_{i}^{I} q_{i j}=1, \quad \int_{Y_{i}} \tilde{P}_{\vec{h}_{i}}(\vec{y}) d \vec{y}=4 \pi
\end{aligned}
$$

were constructed. The parameters of the texure: $G_{k} / L_{k}$ (Gauss/Lorentz part), $g_{k}$ (component position), $b_{k}$ (halfwidth), $F$ (background) and of the pole figures: $n_{j}$ (PF-norm), $q_{i j}$ (PF-part) are given in Table 1. For one component we have $\beta_{k}=55^{\circ}$ and $\gamma_{k}=83.9^{\circ}$ a mostly "unfavourable" orientation for the MPR calculated above. For reconstructing the ODF (all parameters of the table 1 are unknown now) a new approach ${ }^{1}$ was used basing on an optimization by means of standard functions with a direct treatment of the pole figures. All "exerimental" pole figures were calculated for a "thinned" grid of measurement $\left(\Delta \vec{y} \approx 5^{\circ}\right)$ within the range $Y_{i}^{*}: 0^{\circ} \leq \vartheta_{y} \leq 45^{\circ}, 0^{\circ} \leq \varphi_{y} \leq 360^{\circ}$ (Fig. 2). Starting from this data the ODF was determined. All recalculated parameters are given in the table 1 (case refl.). The figures of the recalculated PF are not distinguishable from those of the experimental PF. 


\begin{tabular}{|c|c|c|c|c|}
\hline texture & $g_{k}$ & $b_{k}$ & $G_{k} / L_{k}$ & $F$ \\
\hline model & $\{43.39,55.00,83.90\}$ & $23.00^{\circ}$ & $0.332 / 0.263$ & 0.176 \\
& $\{53.86,90.00,25.90\}$ & $18.00^{\circ}$ & $0.134 / 0.095$ & \\
\hline refl. & $\{43.40,55.00,83.89\}$ & $23.22^{\circ}$ & $0.341 / 0.250$ & 0.186 \\
& $\{53.85,89.99,25.03\}$ & $18.23^{\circ}$ & $0.140 / 0.083$ & \\
\hline trans. & $\{43.37,54.98,83.93\}$ & $23.27^{\circ}$ & $0.344 / 0.244$ & 0.187 \\
& $\{53.84,90.00,25.05\}$ & $18.17^{\circ}$ & $0.138 / 0.087$ & \\
\hline
\end{tabular}

\begin{tabular}{|c|c|c|c|c|c|c|}
\hline pole figures & $n_{1}$ & $n_{2}$ & $n_{3}\left(q_{33}\right)$ & $n_{4}\left(q_{54}\right)$ & $n_{5}$ & $n_{6}$ \\
\hline model & 2220 & 798 & $1540(0.5077)$ & $2184(0.7408)$ & 6848 & 4588 \\
\hline refl. & 2235 & 805 & $1552(0.5084)$ & $2195(0.7403)$ & 6897 & 4626 \\
\hline trans. & 2237 & 804 & $1551(0.5087)$ & $2201(0.7409)$ & 6890 & 4630 \\
\hline
\end{tabular}

Table 1: Parameters of simulated and recalculated ODF and PF

1

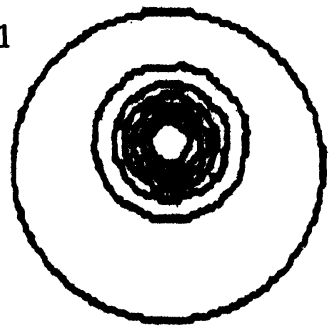

4

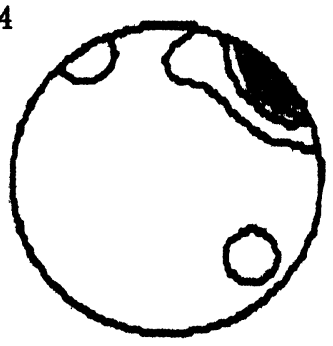

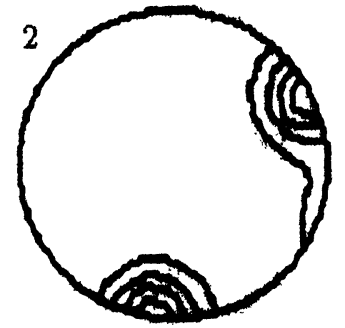

5

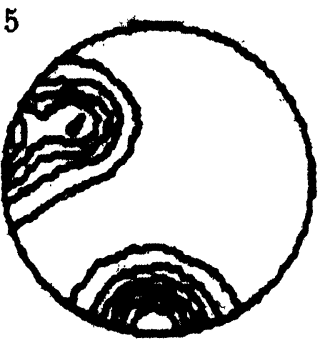

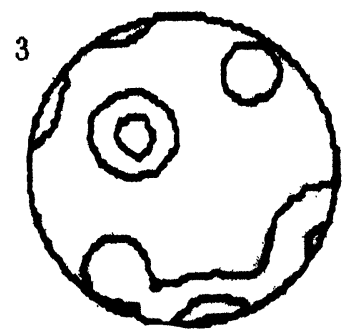

6

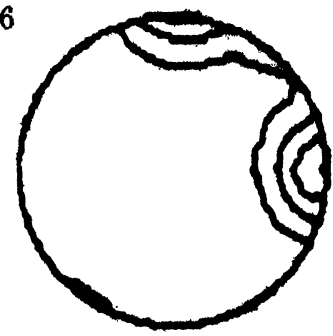

Figure 2: Experimental PF of the modelized sample. Contour lines : 1,2... $Y_{i}^{*}: 0 \leq \vartheta_{y} \leq 45^{\circ}, 0^{\circ} \leq \varphi_{y} \leq 360^{\circ}$. 
Considering the case of transmission measurement $\left(\vartheta_{e}=90^{\circ}\right)$ for MPR we get:

\begin{tabular}{|c|c|c|c|c|c|c|c|c|}
\hline hkl & 021 & 101 & 111 & 120 & 002 & 121 & 130 & 112 \\
\hline$\vartheta_{s}$ & 71.2 & 78.3 & 76.4 & 73.9 & 73.0 & 72.0 & 74.2 & 73.1 \\
\hline
\end{tabular}

The maximal $\vartheta_{s}$ is given by the (021) reflex in the first PF. Starting from PF within the ranges: $Y_{i}^{*}: 70^{\circ} \leq \vartheta_{y} \leq 90^{\circ}, 0^{\circ} \leq \varphi_{y} \leq 360^{\circ}$ we reproduced the ODF described in the Table 1 (case trans.).

\section{Conclusions}

The determination of MPR follows from the necessary condition that every single orientation $g \in G$ must be determinable at least. On the other hand special, sufficient conditions may exist for various reproduction methods (e.g. minimal number of pole figures or pole directions $\vec{y}_{\text {? }}$ ). For this cases MPR may not be sufficient for determining the ODF.

The resolution of the ODF-determination is connected with the density of the measured pole directions $\vec{y}^{5}$. If the maximal angular distance of the measured points $\vec{y}$ is gven by $\Delta \vec{y}$ the attainable resolution is approximately given by $2 \Delta \vec{y}$. For an optimized measurement an equidistant grid is preferred. The smaller the expected halfwidth of the components of the searched ODF the higher the density of the measured grid has to be. Considering this rules ODF's may be determined from MPR with minimal effort. The quality of a result may be tested taking larger and/or more pole figures in a more densed grid of measurement and repeating the ODF-reproduction with this extended pole figure ranges.

\section{Acknowledgement}

Thanks to J.Wilzek and H.Beyer for their help in programing and to A. Vadon and S. Matthies for the suggestion to deal with this problem.

\section{REFERENCES}

1. K. Helming and Th. Eschner, submitted to Crystal Research and Technology

2. K. Helming, Thesis, Akad. Wiss. DDR, ZfK Rossendorf-Dresden (1985)

3. S. Matthies and K. Helming, Phys. Stat. Sol. (b),113, 569 (1982)

4. A. Vadon, Thesis, University of Metz (1981)

5. S. Matthies, G.V. Vinel and K. Helming, Standard Distributions in Texture Analysis, Vol. 1, Akademie-Verlag, Berlin (1987) 